\title{
RESISTENNCIA A ANTIMICROBIANOS EM CEPAS DE Enterococcus spp. ISOLADAS DA UTI DE UM HOSPITAL DE CACHOEIRO DE ITAPEMIRIM - ES
}

\author{
Maicon Marvila Miranda ${ }^{1}$ \\ Ana Carolina Ambrósio Simões ${ }^{2}$ \\ Camilla Dellatorre Teixeira ${ }^{3}$
}

\begin{abstract}
Resumo - A Unidade de Terapia Intensiva, local onde são internados pacientes com maior gravidade, é uma das unidades do hospital com maior índice de infecções hospitalares. Os Enterococcus são capazes também de exibir diferentes mecanismos de resistência adquirida a uma ampla variedade de antimicrobianos. Esta pesquisa teve como objetivo identificar a prevalência de cepas de Enterococcus spp. na UTI de um hospital em Cachoeiro de Itapemirim-ES, no período de 2014 à 2015, e determinar sua multirresistência aos antimicrobianos. Os dados foram coletados através do acervo documental do laboratório de análises clínicas. A maior prevalência da bactéria foi no gênero masculino e em amostras de urina foram fonte majoritária de isolamento. Fora verificado alta taxa de resistência a quinolonas, aminoglicosídeos e glicopeptídeos. A necessidade de uma maior vigilância no controle de infecção hospitalar com o intuito de minimizar e/ou eliminar as infecções de pacientes internados, principalmente em UTI.
\end{abstract}

Palavra chave: Enterococcus spp; Resistência; Antimicrobiano; UTI.

\footnotetext{
1 Mestrando da Faculdade de Medicina da Universidade Federal do Rio de Janeiro/ Departamento de Doenças Infecciosas e Parasitárias, Brasil. E-mail: maiconmiranda@outlook.com.

2 Discente do Curso Bacharelado em Farmácia do Centro Universitário São Camilo, Brasil. E-mail: acasimoes@yahoo.com.br.

3 Orientadora. Farmacêutica. Docente do Centro Universitário São Camilo - ES, Brasil. camilladellatorre@saocamilo-es.br.
} 